\title{
Super resolution laser thermography
}

\author{
by S. Ahmadi*, P. Hirsch*, C. Hassenstein*, P. Burgholzer ${ }^{\star *}$, P. Jung ${ }^{\star \star *}$, G. Caire ${ }^{\star \star \star}$, M. Ziegler* \\ * Bundesanstalt für Materialforschung und -prüfung (BAM), 12200 Berlin, Germany, samim.ahmadi@bam.de \\ ${ }^{* *}$ RECENDT, Research Center for Non-Destructive Testing, Linz, Austria \\ *** Technische Universität Berlin, Berlin, Germany
}

\begin{abstract}
In order to break the thermographic spatial resolution limit (super resolution), the combination of spatially structured heating and numerical methods of compressed sensing can be used.

Our studies deal with the influence of experimental parameters such as the pulse length of the laser illumination and the size of the laser spot. Furthermore, we have dealt with the choice of parameters in the reconstruction that have an influence on the underlying minimization problem in terms of compressed sensing. Finally, the results of the super resolution method are compared with the results based on conventional thermographic testing methods.
\end{abstract}

\section{Introduction}

Thermographic NDT is based on the interaction of thermal waves with inhomogeneities. The propagation of thermal waves from the heat source to the inhomogeneity and to the detection surface according to the thermal diffusion equation leads to the fact that two closely spaced defects may be incorrectly detected as one defect in the measured thermogram. This can be circumvented by using super resolution techniques such as the blind structured illumination in combination with an approach from mathematical optimization theory and compressed sensing. Such compressed sensing algorithms are useful within sparse systems since they make use of joint sparsity [1]. In our thermal images the sparsity is given by the small number of defects or more precisely by the small relation between number of pixels which are in the defect area and number of pixels in the non-defect area.

Essentially, our studies are concerned with analyzing and evaluating the potential of new super resolution techniques within thermography. We vary the experimental design as well as the choice of regularization parameters in post-processing. In addition, we show complementary techniques that allow the potential of super resolution techniques to be further exploited.

\section{The implementation of super resolution techniques in thermography}

Our super resolution studies rely on thermographic data which is generated by structured heating. Following the Green's function ansatz, the resulting temperature field in transmission configuration can be described by the 1D solution for a line source as follows [2]:

$$
T(x, z=L, t)=T_{0}+\frac{2}{\rho c_{p} \pi 4 \alpha} \int_{0}^{t} \int_{-\infty}^{\infty} q(x-\tilde{x}, t-\tilde{t}) e^{-\frac{(x-\tilde{x})^{2}}{4 \alpha(t-\tilde{t})}} \sum_{n=1}^{\infty} R^{2(n-1)} e^{-\frac{(2 n-1)^{2} L^{2}}{4 \alpha(t-\tilde{t})}} \frac{d \tilde{t}}{\tilde{t}} d \tilde{x}
$$

$T_{0}$ stands for the initial temperature, $\rho$ for the mass density, $c_{p}$ for the specific heat, $\alpha$ for the thermal diffusivity, $q$ for the heat flux density, $R$ for the thermal reflectance from the material to air, and $L$ for the thickness of the specimen. Moreover, Equation (1) shows that the temperature distribution depends on the pulse length (convolution in time with variable $\tilde{t}$ ) and on the laser line width (convolution in space with the variable $\tilde{x}$ ). The heat flux density $q$ is given by the element-wise product of the temporally and spatially dependent irradiance distribution of the laser and the spatially varying absorptance pattern of the sample. This equation can be interpreted as the convolution of a thermographic point spread function (PSF), with the absorptance related heat source.

\subsection{Experiment with structured heating}

The experiment has been performed using a high-power laser with a linear spot shape. The basic measurement strategy is shown in figure 1. We worked in transmission configuration and investigated a metallic specimen with blackened line pairs of varying distance at the illuminated side of the otherwise untreated surface. These black line pairs have a much higher absorptance as compared to the rest of the surface and when illuminated by the laser lead to a much higher heat flux density. Therefore, these absorption lines represent the defects that we normally try to detect by using thermographic NDT. We used this simple thermographic NDT scheme in order study novel super resolution techniques and to super 
resolve these lines with a distance that is much below the classical limit. In our experiments we studied the influence of the experimental (e.g., the laser line width and the pulse length) and numerical parameters on the super resolution power.

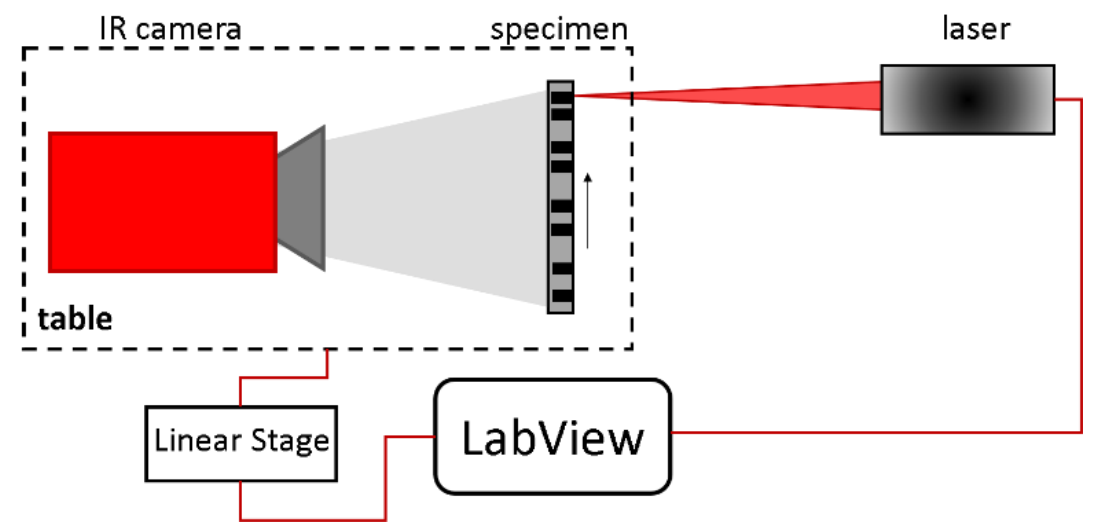

Fig. 1. Experimental setup: laser scanning thermography; the whole table is moved by a linear stage which is controlled by a computer with LabView. The laser can be used in step scanning mode (temporal sequence: 1. Laser pulse, 2. Keep the laser turned off while moving the specimen for a certain distance and let the specimen cool down, again, 1. etc.) or in continuous scanning mode (move the specimen constantly with a certain velocity while the laser is turned on continuously)

\subsection{Post-processing using compressed sensing based algorithms}

Compressed sensing based algorithms in thermography rely on the solution of the general forward problem, which can be written as a simple but underdetermined matrix equation [3]:

$$
\boldsymbol{A} \cdot \boldsymbol{x}=\boldsymbol{y}
$$

whereby in our scenario these variables can be interpreted for each frame or measurement as: $\boldsymbol{A}$ refers to the convolution matrix of the thermographic point spread function (PSF; in the step scanning case the PSF can be taken from equation (1)), $\boldsymbol{x}$ refers to the heat flux density induced by the varying spatial overlap of the defect pattern and the total set of all the different illuminations and $\boldsymbol{y}$ stands for the measurement data (i.e., many different thermographic sequences). Based on this simple equation, there are a lot of optimization algorithms that find a solution for $\boldsymbol{x}$ for given $\boldsymbol{A}$ and $\boldsymbol{y}$ such that $\boldsymbol{A} \cdot \boldsymbol{x}-\boldsymbol{y} \rightarrow 0$. The basic idea behind compressed sensing is to use the sparsity inherent in the measurement signal ensemble by a mathematical optimization process. Sparsity enters the equation in form of a L1 regularization term. The iterative joint sparsity approach IJOSP $[1,4]$ is also minimizing this difference but considers the joint sparsity for all measurements, which means it only leads to a nonzero entry in the solution if several measurements show a signal at the same position. These positions are then identified as the defect positions.

The reconstruction goodness, i.e. the probability to detect and to super resolve the closely separated defect pairs, correlates with the number of measurements performed. For our case, usually a total number of measurements on the order of $O\left(10^{2} \ldots 10^{3}\right)$. The dimension of problem (2) is accordingly very high and it is advisable to reduce it by using some intermediate thermographic reconstruction step [5]. 


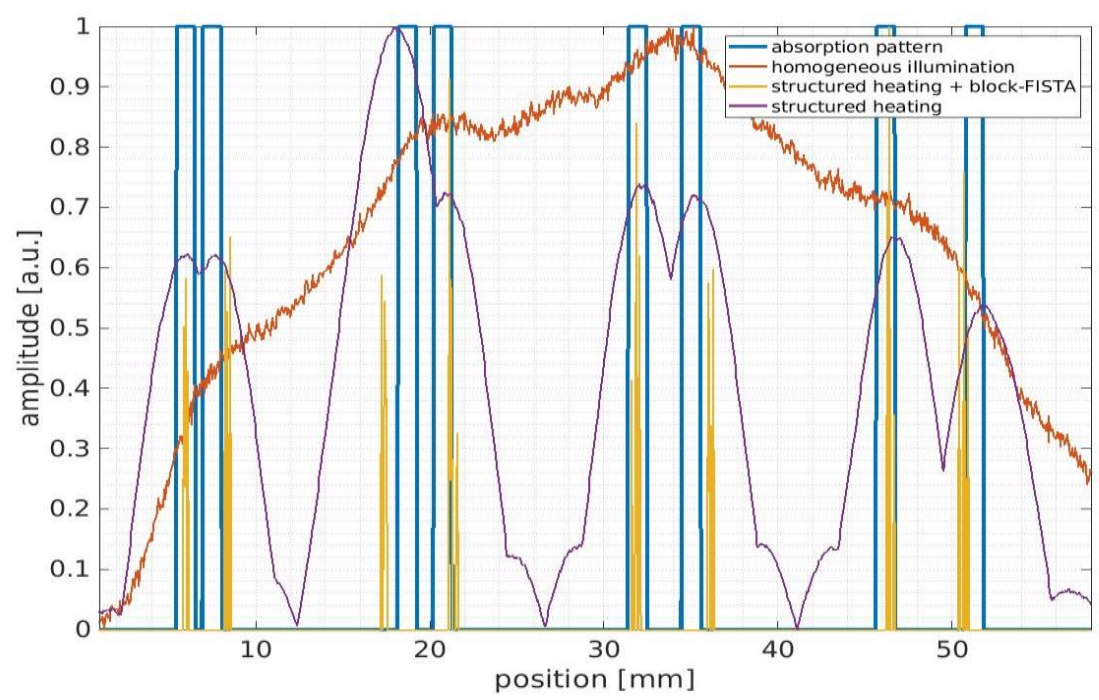

Fig. 2. Comparison of different reconstruction techniques: homogeneous illumination and structured heating refers to the maximum thermogram of these measurements, respectively; the absorption pattern represents the absorption lines on the front surface of the specimen. Structured heating has been realized by using a laser line width of $w=1.5 \mathrm{~mm}$ and a pulse length of $t_{\text {pulse }}=500 \mathrm{~ms}$

\section{Results and outlook}

Figure 2 shows some results for using structured illumination with a laser line width of $1.5 \mathrm{~mm}$ (step scanning mode), the conventional way of homogeneous illumination (illuminating the whole surface of the specimen) and applying the IJOSP algorithm which is based on block-FISTA on the results from structured illumination thermography.

It can be clearly seen that the application of a compressed sensing based algorithm such as the IJOSP approach, which uses the block-FISTA for optimization, highly improves the detection sensitivity and delivers clear peaks, although sometimes associated with uncertainties regarding the position. This interesting result could already be seen in [1,5] using high-power VCSEL arrays in a steady state mode. Our studies show that this super resolution technique can also be applied on thermographic data from laser line scanning which is of high interest for industrial applications and therefore provides a solution to enhance the spatial resolution.

Instead of using the block-FISTA optimization with the given data set, some further signal processing steps that optimize the incoherence of the measured data can be done for the benefit of joint sparsity which are also discussed in our studies. Moreover, the influence of the experimental design as well as the choice of regularization parameters have been evaluated: The higher the laser line width the worse the reconstruction quality in super resolution thermography. The same is valid for the laser pulse length in pulsed thermography measurements, but the variation of the laser pulse length has not such a high degrading effect on the reconstruction quality than the variation of the laser line width. However, the highest effect on the reconstruction quality is given by the variation of the regularization parameters in our compressed sensing based methods, e.g. in the block-FISTA optimization where the step size for optimization as well as a denoising parameter can be chosen. Due to the high impact of the choice of the regularization parameters, we are developing a new deep learning based approach for block sparsity optimization in thermal imaging.

\section{REFERENCES}

[1] P. Burgholzer, T. Berer, M. Ziegler, E. Thiel, S. Ahmadi, J. Gruber, G. Mayr \& G. Hendorfer, Blind structured illumination as excitation for super-resolution photothermal radiometry, Quantitative InfraRed Thermography Journal, 2019.

[2] K. D. Cole et al., Heat Conduction Using Green's Functions, 2 ${ }^{\text {nd }}$ Edition, CRC Press, 2010.

[3] E. J. Candes et al., Stable signal recovery from incomplete and inaccurate measurements, Comm. Pure Appl. Math. 59, 2005.

[4] P. Burgholzer et al., Super-resolution thermographic imaging using blind structured illumination, Applied Physics Letters 111 (3), 2017.

[5] S. Ahmadi, P. Burgholzer, G. Mayr, P. Jung, G. Caire \& M. Ziegler, Photothermal super resolution imaging: A comparison of different thermographic reconstruction techniques, submitted in NDT \& $E$ International (under review), 2019. 